Revista Digital Año 7. No 9 - Año 2016. --pág. 1-104

ISSN 1853-1393

Resistencia. Chaco. Argentina - 2016

\title{
LA FORMACIÓN DOCENTE Y EL EJERCICIO PROFESIONAL DE LOS NOVELES PROFESORES EN HISTORIA
}

THE TEACHING TRAINING AND THE PROFESSIONAL EXERCISE OF NOVELS TEACHERS IN HISTORY

Prof. Irma Esther Rosso ${ }^{1}$ - Mg. María Teresa Alcalá ${ }^{2}$

Fecha de recepción: 12-08-2016

Fecha de aceptación y versión final: 17-10-2016

Resumen: El ejercicio de la docencia se considera como un proceso continuo desde la etapa de estudiante de la carrera del profesorado en Historia hasta la inserción laboral. Es decir, que la universidad avala la formación disciplinar de sus egresados y los acredita para la práctica profesional. Situación cierta, aunque, en la realidad, el inicio de la docencia, significa enfrentarse a una serie de problemas generalmente no abordados en la institución de formación docente.

Mediante relatos de docentes noveles analizamos situaciones referidas a: temores, inseguridades, incertidumbres que experimentan al transitar esta etapa inicial del ejercicio profesional.

Para aproximarnos a las vicisitudes que vivencian y marcan a estos docentes indagamos sus procesos de socialización profesional y de conformación del conocimiento profesional a través de la investigación biográfica y narrativa.

Palabras clave: Profesores Noveles - Inserción profesional - Socialización Profesional - Modelos de expertos

Abstract: The exercise of teaching is considered as a continuous process from the student stage of the career of teachers in History to the insertion of labor. That is, the university endorses the disciplinary training of its graduates and accredits them for professional practice. True, although, in reality, the beginning of teaching, means facing a series of problems not usually addressed in the institution of teacher training.

Through stories from novice teachers, we analyze situations related to: fears, uncertainties, uncertainties that they experience during this initial stage of the professional exercise.

In order to approach the vicissitudes that live and mark these teachers, we investigate their processes of professional socialization and the formation of professional knowledge through biographical and narrative research.

Key words: New Professors - Professional insertion - Professional socialization - Models of experts

\section{Introducción}

En este artículo abordamos las vivencias de docentes principiantes que permiten identificar aspectos críticos de la formación inicial y de la etapa de inserción en la docencia que se consideran fundamentales para un desarrollo profesional comprometido con la promoción de aprendizajes escolares relevantes y potentes. En este sentido, nos ubicamos en la línea de investigación didáctica que estudia la construcción del conocimiento profesional docente focalizada en la etapa de iniciación (docentes principiantes o noveles).

\section{Orígenes de los estudios acerca del conocimiento profesional docente (CPD)}

Las investigaciones en el campo de la Didáctica referidas al pensamiento y al conocimiento del profesor tienen fundamentos epistemológicos diferentes. En su origen, la psicología conductista ofreció las bases conceptuales y metodológicas para

\footnotetext{
${ }^{1}$ Instituto de Ciencias de la Educación. Facultad de Humanidades. Universidad Nacional del Nordeste.

2 Instituto de Ciencias de la Educación. Facultad de Humanidades. Universidad Nacional del Nordeste.
} 
analizar los procesos cognitivos y de acción del docente vinculados con los aprendizajes de los estudiantes (Ortega Iglesias y Perafán Echeverri, 2012). En este sentido, Schulman (1989) identifica al programa proceso-producto como el modelo dominante de las investigaciones acerca de la enseñanza eficaz entre la década del cuarenta y la del setenta, sustentado en una concepción simple de los procesos didácticos que acontecen en las aulas reales, tal como lo indica Pérez Gómez (1992:82): "La vida del aula puede reducirse a las relaciones que se establecen entre el comportamiento observable del profesor/a cuando enseña y el rendimiento académico del alumno/a".

Hacia los setenta este paradigma va a ser fuertemente cuestionado por las siguientes razones: el reduccionismo del análisis que propicia acerca de la práctica pedagógica, la relación causal/lineal que supone entre los procesos de enseñanza y aprendizaje; la descontextualización de los comportamientos de docentes y estudiantes; la rigidez de los instrumentos de observación; la desconsideración del papel de los contenidos en la relación didáctica y la pobreza conceptual del programa en sí mismo. Además, los desarrollos de la ciencias cognitivas y la pluralidad metodológica que las ciencias sociales van a ir instalando respecto de la naturaleza compleja, histórica y situada de los fenómenos sociales, incidirán en el surgimiento de programas de investigación que pudieran proporcionar categorías conceptuales alternativas. De este modo, los estudios sobre el pensamiento del profesor asumirán dos presupuestos: "(... primero, los profesores toman decisiones racionales adaptadas a un contexto complejo e incierto; segundo, los pensamientos, juicios y decisiones del profesor guían su comportamiento" (Ortega Iglesias y Perafán Echeverri, 2012: 19). En esta línea se puede distinguir a la vez, dos enfoques característicos, el cognitivo y el alternativo.

El enfoque cognitivo representado por Clark y Peterson (1990), Calderhead (1996), entre otros, focalizó el análisis del pensamiento del profesor en la planificación (pensamientos preactivos y postactivos); el pensamiento durante la acción didáctica (pensamientos y decisiones interactivos); y el análisis de las teorías y creencias de los profesores que constituyen la base de las decisiones que toma y la perspectiva desde donde interpreta los acontecimientos del aula.

Ortega Iglesias y Perafán Echeverri (2012) señalan que este enfoque fue objeto de críticas en algunas categorías interpretativas (pensamiento durante la planificación docente y pensamientos y decisiones interactivos), en función de "cierta inclinación por indagar aspectos predictivos en el marco de la enseñanza efectiva, propio del programa proceso-producto" (20-21).

El enfoque alternativo se erige sobre la base de una conceptualización diferente del proceso de enseñanza. Ya no se examina la enseñanza en el sentido de una acción eficaz, sino que se la vincula a las intencionalidades del docente y a la cultura que lo constituye. Por lo tanto, la intencionalidad de la enseñanza resulta fundamental para comprender el accionar del profesor así como las epistemologías que ha construido y que componen su conocimiento.

Desde esta concepción se complejiza la enseñanza y se requiere de una diversificación metodológica que pueda dar cuenta de tal complejidad. Advertimos que la enseñanza pasa de ser considerada una "tejné" a ser concebida como "praxis", es decir, como "acción informada", "acción que se crea", donde hay reflexión en y sobre la acción, que modifica la base de conocimientos y la acción misma.

Perafán (2004) reconoce tres campos de estudios alternativos: a) el conocimiento profesional del profesor como categoría general; b) las epistemologías del profesor sobre su propio conocimiento profesional, y c) el conocimiento profesional específico del profesorado asociado a categorías particulares.

En cuanto al CPD como categoría general, las investigaciones muestran un rico y variado abanico de categorías y componentes con los que se intenta atrapar su 
naturaleza y proceso de elaboración inicial y continua. Los diversos estudios coinciden en que se trata de un conocimiento práctico, epistemológicamente diferenciado y distinto a otros tipos de conocimientos profesionales. Su construcción es gradual y progresiva, a partir de las concepciones de los profesores, sus obstáculos y sus posibles hipótesis de progresión que facilitan su evolución (Porlán y Rivero, 1998).

\section{El proceso de construcción del conocimiento profesional docente (CPD)}

La elaboración del CPD comienza mucho antes de que el sujeto se forme sistemáticamente para el ejercicio profesional. Desde que ingresa en el sistema educativo, como estudiante, inicia un proceso de enculturación en esa cultura particular que es la escolar, en función de la cual construye concepciones, teorías, creencias respecto de qué es ser docente, qué y cómo enseñar, qué y cómo aprender, qué es el conocimiento, para qué sirve la escuela, etc.

Se trata de un proceso de construcción constituido por trayectos de formación que se realizan a través de dos vías, una asistemática y más vivencial, y otra explícita, sistemática, organizada. La primera, incluye la biografía escolar y la socialización profesional de los sujetos. En el segundo trayecto de formación, se reconocen tres etapas: la inicial, la de inserción y la de desarrollo profesional.

La etapa inicial supone el "primer punto de acceso al desarrollo profesional continuo" (Marcelo y Vaillant, 2009:48). Ésta es una etapa fundamental, pero muy criticada y devaluada, que precisa un profundo replanteo y transformación en todos sus aspectos: curriculares, organizativos y personales.

La etapa de inserción profesional es "(...) la transición desde profesor en formación hasta llegar a ser un profesional autónomo. La inserción se puede entender mejor como parte de un continuo en el proceso de desarrollo profesional de los profesores" (Vonk, en Marcelo y Vaillant, 2009:49). Esta etapa corresponde a la del profesor principiante o novel en la que básicamente debe enseñar y aprender a enseñar, sobre la cual hacemos foco en el presente artículo.

El desarrollo profesional, es un fenómeno multidimensional y complejo referido al carácter continuo del aprendizaje, así como a su enraizamiento en las vidas de los individuos (Kelchtermans, en Marcelo y Vaillant, 2009: 77).

Ahora bien, ¿qué tipo de conocimiento se elabora en los espacios de formación docente y de desarrollo profesional? Se trata de un conocimiento profesional: "conocimiento práctico, (que) no se encuentra separado del conocimiento teórico adquirido previamente, sino que, en realidad, aparece como un conocimiento transformado en razón de la acción práctica del docente, de su experiencia personal" (Alcalá, Núñez y Demuth, 2012:3).

\section{¿Cómo se aprende un conocimiento que integra y articula teoría y práctica?}

EI CPD se construye en la práctica y en la reflexión sobre esa práctica, en la que está implicado el sujeto. Para ello es preciso aprender cómo aprender de la práctica (Marcelo Vaillant, 2009:110), ya que la acción deviene en fuente de aprendizaje si media una voluntad consciente y explícitamente orientada hacia la objetivación de la acción, y esto se aprende, no se da de manera natural, se requiere de dispositivos de formación especialmente elaborados para tal fin.

En la línea de pensamiento que exponemos, concebimos a la formación docente como un trayecto de construcción y desarrollo del CPD, en sentido amplio es un proceso que abarca toda la vida profesional debido a que el/la profesional de la docencia construye y re-construye su saber específico de manera permanente.

Gorodokin (2006) define a la formación docente como el:

(...) proceso en el que se articulan prácticas de enseñanza y de aprendizaje orientados a la configuración de sujetos docentes/enseñantes. La práctica docente se 
concibe en un doble sentido: como práctica de enseñanza, propia de cualquier proceso formativo y como apropiación del oficio del docente, como iniciarse, perfeccionarse y/o actualizarse en la práctica de enseñanza. (4)

Desde esta perspectiva, surge entonces otro interrogante referido a los procesos de formación docente: ¿Qué la relación con el saber que se promueve?, puesto que los profesores terminan enseñando no lo que saben sino cómo lo saben:

"(...) Al enseñar, uno se expone, se enseña; no sólo enseña un saber, sino la propia relación con el saber (...) es ante todo presencia. $Y$ esto es lo que primero perciben alumnos y alumnas: la presencia (o la ausencia), el modo de ser alguien que se muestra (o no) y entabla (o no) una relación, tanto con el propio alumnado, como con lo que pretende enseñar, o compartir, o estimular e impulsar en esa relación personal. Hacerse docente tiene mucho que ver con elaborar esa presencia (...)" (Contreras Domingo, 2010: 64).

La formación docente basada en la transmisión (en el sentido de trasvasamiento de teorías descontextualizadas) se caracteriza por la separación entre conocimiento y sujeto. El conocimiento como proceso de transformación personal no tiene lugar porque, al igual que a los objetos, "se lo posee". Esta concepción es un emergente cultural del capitalismo, de la "sociedad del conocimiento", en la que las ideas circulan objetivadas, se usan, son externas a los sujetos que "hacen cosas" con ellas.

Para que la formación se convierta en experiencia tiene que brindar oportunidades para pensar-se a sí mismo, como fuente de saber y de ser; para acceder a otras formas de saber y de relación con el saber: el saber in-corporado, que nos constituye y necesitamos para vivir. La consecuencia de esta concepción de conocimiento y de relación con el saber es lo que con acierto afirma Contreras Domingo (2010): "Formarse como docentes es necesariamente hacer algo consigo mismo" (66).

Por otra parte, es preciso reconocer que la formación inicial no puede reproducir los contexto reales del ejercicio profesional como ocurre en todas las carreras que preparan para desempeños profesionales, sin embargo, las investigaciones han confirmado y continúan corroborando que los futuros docentes deben aprender en la práctica (Feiman, 2001). Aunque, como señalan Ball y Cohen (1999), "estar centrado en la práctica no necesariamente implica situaciones en las aulas en tiempo real" (14).

Al respecto, Carlos Marcelo (2008) aclara lo siguiente: "para aprender los profesores necesitan utilizar ejemplos prácticos, materiales como casos escritos, casos multimedia, observaciones de enseñanza, diarios de profesores y ejemplos de tareas de los alumnos" (23). Esto podría propiciar actividades de análisis y reflexión sobre la enseñanza real.

\section{La opción metodológica: las narrativas de los/las noveles profesores en Historia}

Nuestro principal interés es indagar el conocimiento práctico-personal que construyen los noveles profesores en historia en tanto constituye el conocimiento propio y particular de la profesión docente. Este interés impone un abordaje metodológico que potencialmente ofrezca los criterios, estrategias e instrumentos que se adecuen a las características particulares del CPD, tal como lo expresa Bolívar (2005):

Tras la crisis del positivismo, dentro del marco hermenéutico, han aflorado una pluralidad de perspectivas, agudizadas con la crisis de la modernidad. Desde estas metodologías alternativas (práctica reflexiva, narrativa, hermenéutica, vida de profesores, investigación del profesor, etc.) se ha pasado de la necesidad de un conocimiento para la enseñanza (producido por la investigación de expertos externos para prescribir a las aulas), a reconocer un estatus propio al conocimiento del profesor; 
de un conocimiento formal-teórico, procedente de la investigación, a un conocimiento práctico-personal que poseen los profesores. (3)

También, los objetivos del estudio privilegian dos aspectos que consideramos centrales: las concepciones epistemológicas de los docentes noveles o principiantes (referidas a la concepción de la historia como ciencia y sus enfoques), y las propuestas didácticas que formulan, por lo que la metodología interpretativa con una perspectiva narrativa nos proporciona las herramientas más adecuadas para comprender la práctica de los sujetos indagados.

En este punto resulta imprescindible señalar que la perspectiva mencionada implica mucho más que una metodología de recogida de datos, tal como lo expresan Álvarez y Porta (2012) cuando identifican los supuestos del enfoque biográficonarrativo:

Es narrativo, por cuanto la práctica y el conocimiento experiencial son representados por vía narrativa; es constructivista, en tanto el pasado se explica, se construye e interpreta con referencia al presente; es contextual, ya que las historias sólo tienen sentido en sus contextos sociales, culturales e institucionales; es interaccionista, por cuanto los significados se construyen e interpretan (individuo y contexto), y las fuentes y actores no tienen existencia "real", sino que son construidos en/con los relatos. Por último, es dinámico, en tanto proceso con dimensiones temporales (81).

Desde la perspectiva que asumimos entonces, los relatos de los noveles profesores permiten dar cuenta de los contextos sociales, culturales y políticos (Rivas Flores, 2009) de su formación, en particular, el contexto de la Facultad de Humanidades de la UNNE donde realizaron la etapa de formación inicial, así como el de las instituciones donde comenzaron su práctica profesional docente.

Dado que nuestro foco son las concepciones epistemológicas y las propuestas de enseñanza de los principiantes, cabe aclarar que consideramos al curriculum como texto y contexto de los procesos formativos de los sujetos en estudio. El curriculum regula la acción docente y las interacciones didácticas, pero también los docentes en tanto intérpretes mediadores del curriculum, con sus modos de enseñar, de trabajar los contenidos y de evaluar, y con los materiales que seleccionan y utilizan, contribuyen al desarrollo de los procesos de construcción del conocimiento práctico y concepciones disciplinares de los futuros profesores. En otras palabras, el curriculum tal como lo configuran los actores que lo realizan en la práctica, constituye ámbito y contenido de aprendizaje del profesional docente.

En este sentido, los relatos de las principiantes entrevistadas son reconstrucciones de experiencias y reflexiones en torno a su propia práctica y a los procesos formativos que vivieron en la institución formadora y en las que han iniciado su trabajo docente. Tales reconstrucciones, como a continuación mostramos, dan cuenta de las vicisitudes características de la iniciación en la docencia y los problemas de su inserción relevados en investigaciones internacionales (Marcelo, 2007).

Los docentes nóveles: su inserción y desarrollo en el campo profesional docente

Consideramos docente novel o novato (Alliaud, 2004), o principiante (Marcelo, 2008), a sujetos con no más de tres o cinco años de antigüedad en la tarea de enseñar.

Para aproximarnos a las características y problemáticas específicas propias de esta etapa de inserción y primeros desempeños profesionales docentes, analizamos los relatos autobiográficos de profesores en historia referidos a los procesos de socialización y conformación del CPD, intentando identificar elementos o aspectos que sustentan su identidad profesional (Marcelo y Vaillant, 2009). Identidad que se construye individual y colectivamente, en una trama intersubjetiva de relaciones con colegas, alumnos, instituciones y formas de actuar cotidianas, que generan prácticas, las que a través del tiempo quedan arraigadas en la comunidad educativa y se 
transforman en normas consuetudinarias incuestionables que se deben cumplir. Cada institución cuenta con un cúmulo de estas normas que difieren, según los rasgos de ésta, las que son acatadas por el o la principiante (Marcelo, 2008).

En relación con lo anterior, los principiantes tienen que aprender el oficio y la cultura docente, y ambos aprendizajes dependen también de la personalidad del sujeto y de su capacidad de adaptación al entorno social e institucional al que comienza a integrarse. Al respecto, autores como Veeman (1984) resaltan la conflictividad inherente a esta etapa inicial con el concepto de "choque con la realidad". Durante el primer año de ejercicio de la docencia, la mayoría de los sujetos realizan intensos aprendizajes del tipo ensayo-error que se caracteriza por un predominio del valor práctico y de un principio de supervivencia (Marcelo, 2007).

En general, el ejercicio inicial de la docencia es concebido como un continuum entre el ser estudiante de la carrera de formación docente -en este caso, del profesorado en Historia-, y la inserción laboral con características distintivas y determinantes del desarrollo profesional evolutivo (Marcelo, 2007). La universidad avala la formación en la disciplina de sus egresados y los acredita para la práctica profesional de la docencia. No obstante, en la mayoría de las instituciones que ofrecen profesorados en diferentes disciplinas científicas, el imaginario de una formación para el ejercicio profesional no condice con las vivencias de los docentes principiantes al hacerse cargo de cursos en las instituciones educativas del nivel secundario, de acuerdo con lo que los sujetos estudiados expresan en las narraciones respecto de su desempeño durante las primeras experiencias de enseñanza en las instituciones escolares clases.

En sus autobiografías los docentes noveles dan cuenta de una práctica inicial que los hizo enfrentarse a "problemas" concretos, habitualmente no abordados en la institución formadora, experiencia que constituye el "choque de realidad" anteriormente referido, tal como lo relata Adriana:

La Formación Profesional que tuve me brindó muchísimos ejemplos a seguir..., que fue forjando mi propia personalidad profesional, ... que se desmoronaron el primer día que estuve frente a un curso, ... (En ese) primer día de profesora de Historia, sola, con 45 alumnos en un $1^{\circ}$ año no me sirvieron ninguna de las herramientas que yo pensé que poseía.

Las expresiones de la principiante respecto de la inutilidad de las herramientas adquiridas en su trayecto formativo para la práctica docente se reiteran en los relatos autobiográficos referidos a esta etapa de transición del rol de estudiante al rol docente en contextos situados y complejos.

La capacidad de adaptación y de supervivencia se pone en juego y de esto depende la posibilidad de convertirse en experto o experimentado, tal como observamos en las palabras de Soledad:

Hoy como profesora de un $8^{\circ}$ año de una escuela oficial urbana con distintos chicos de distintas edades y niveles de vida diferentes debo empezar a enseñarles a escribir a interpretar consignas etc, es por eso que siempre realizo cursos de formación ética, geografía y sicología ya que todo lo que nos enseñaron no nos sirve, se dedican a dar temas que cuando los volcamos a la realidad no tienen nada que ver, no estamos capacitados para tantas cosas a la vez, no es nada fácil, los alumnos vienen con problemas de escritura o de leer de años anteriores. En un octavo tenés que retomar todo, porque los de noveno exige (sic) y la de lengua no sabe qué hacer, y algunos padres ni se preocupan por ello, y otras no quieren que tengan baja las notas, ¿qué debemos hacer? Debo ser expositiva por más que quiera ser constructivista en todas mis clases. 
A pesar de todos los sinsabores, amo la docencia aprecio mucho a mis alumnos ya que ellos me necesitan.

La profesora de sicología cuando nos dicta los cursos, materia que me gusta muchísimo, nos dice que al dar la clase debo tener en cuenta cómo doy esa clase, debemos dar cariño y estima, para que ellos aprecien la materia o al menos lograr que les guste, no olvidemos que son personitas que tienen dificultades y sentimientos como yo, y no debo olvidar que estoy formando ciudadanos que están insertados en una sociedad tan cambiante con tantos aspectos negativos más que positivos, si uno enseña que son valores, ellos lo ven día a día que esos valores no tienen en cuenta, cuestionan y una debe estar preparado y buscar dentro de lo posible la respuesta más correcta.

Por eso quiero llegar a ser una excelente profesora, no digo erudita, pero tener muchos conocimientos, una biblioteca con libros suficientes para poder ser buena docente constructivista y tomar del conductismo para hacerla innovadora, en todos los aspectos, para que los demás tengan mayor aprehensión de la materia, ya que la realidad social, nos exige.

Advertimos en el testimonio de Soledad no solo el reclamo por las carencias que identifica en su formación inicial, sino también el "motor" que le permite continuar en una senda de construcción de la experticia docente.

Convertirse en un buen profesor implica un largo y complejo recorrido. La categoría de profesor experto aparece como opuesta a la de profesor novel o novato o principiante. Conocer el pensamiento y actuación de ambos tipos constituye una de las líneas de investigación más fértiles en el campo actual de la Didáctica en orden a la elaboración de principios y propuestas de formación docente más adecuadas a las demandas de la educación en los tiempos que corren.

Por ejemplo, a partir de sus estudios Berliner y colaboradores (en Angulo Rasco, 1999:284), han identificado características que diferencian a un experto de un novel. Los docentes expertos poseen estructuras de conocimiento más elaboradas de las experiencias de clases pasadas, que les permiten comprender con mayor profundidad, riqueza y aún rapidez, las tareas de enseñanza y los acontecimientos de clase, además de poder llegar a predecir con bastante exactitud lo que va a ocurrir en el aula. Esto es así porque los profesores expertos conocen las situaciones del aula, desarrollan repetidas rutinas para controlar la clase y explicar e interpretar automáticamente los acontecimientos que tienen lugar en ella y actuar en consecuencia.

No obstante, el conocimiento rutinario puede obstaculizar los esfuerzos de los profesores por reflexionar sobre sus propias prácticas, por ver las cosas desde una nueva perspectiva o aprender nuevos enfoques de la enseñanza (Putnam y Borko, 2000). Aludimos aquí al riesgo del docente experto rutinario al que se refieren Bransford, Derry, Berliner y Hammersness (2005), el que si bien logra eficiencia mediante el desarrollo de un conjunto de competencias que aplica a lo largo de vida profesional, no puede ampliarlas ni profundizarlas como lo hacen los docentes expertos adaptativos.

En referencia al aporte de los autores arriba citados, Marcelo (2008), señala la relevancia de dos dimensiones en procesos de convertirse en profesor experto: innovación y eficiencia.

La investigación muestra que la gente se beneficia más de oportunidades de aprendizaje que hacen balance entre las dos dimensiones dentro del corredor de desarrollo óptimo... En el caso de los profesores principiantes, la dimensión eficiencia juega un papel psicológico importante. En cualquier área de conocimiento o nivel, los profesores principiantes a menudo quieren instrucciones paso a paso de cómo hacer las cosas de modo eficiente... En general están muy preocupados por los "cómo" y menos por los porqué y los cuándo. Aunque esta dimensión procedimental sea 
importante, la investigación nos muestra que por sí sola no conduce un desarrollo docente eficaz, a menos que se acompañe por la dimensión innovación que representa la necesidad de ir más allá de las habilidades orientadas a la eficiencia y adaptarse a las nuevas situaciones (14).

Retomando las diferencias entre docentes expertos y noveles, Putnam y Borko (2000) señalan que los profesores que poseen una comprensión más rica de la materia de sus asignaturas tienden a poner de relieve en sus asignaturas los aspectos conceptuales, la resolución de problemas y la indagación, por el contrario, los profesores menos conocedores de la materia tienden a poner de relieve los hechos y los procedimientos.

La noción de profesor experto entraña, además de los componentes cognitivos señalados, aspectos relacionados con la reflexión crítica, el compromiso con el aprendizaje de los estudiantes y concepciones complejas de conocimiento.

La experticia supone fundamentalmente buena enseñanza, tanto por los logros de aprendizaje de los alumnos como la calidad de qué se enseña (relevancia y profundidad de los contenidos) y de cómo se lo hace (idoneidad práctica). De esta manera, experticia no es sinónimo de "antigüedad docente", si bien el tiempo de desempeño profesional es una condición indispensable, no es suficiente para distinguir a un docente experto.

Arribamos así a una cuestión central referida a las culturas institucionales y a la organización escolar en la medida que explícitamente ofrezcan alternativas de acompañamiento a los noveles o "recién llegados" al campo profesional por un lado, y por otro, espacios y dispositivos de formación permanente de los docentes experimentados.

En relación con el acompañamiento de los principiantes aludimos a que las escuelas desarrollen una "cultura de inserción" (Marcelo, 2007), es decir, que existan estrategias y disposiciones personales para su orientación y apoyo durante la etapa de iniciación profesional caracterizada por la desorientación, el miedo, el estrés por la sobrecarga de tareas para las que no se sienten totalmente preparados. El relato de Miriam que transcribimos seguidamente ilustra las vivencias de los noveles al respecto:

Empecé a ejercer la docencia, primero como suplente, con muy pocas horas, con mucha incertidumbre, expectativas, miedos y a la vez muchas ganas. Muchas veces me sentía institucionalmente "descolgada" a raíz del poco tiempo que estaba en la escuela.

Fue pasando el tiempo, con algunas que otras angustias, decepciones y también alegrías. A medida que fui incrementando horas me fui sintiendo mejor, más segura, más confiada. Siempre conté con directores que sobre todas las cosas, supieron esperarme, confiaron en mí, me apoyaron, me ayudaron a crecer profesionalmente y también me enseñaron con sus actitudes, ejemplos, consejo.....También rescato la tarea de colegas de los cuales he aprendido.-.

Es interesante el aporte que nos hace Miriam, ella se identifica de la misma manera que lo hacen la mayoría de los profesores noveles, comienzan con incertidumbre, miedos, es más, aclara que al principio se sentía "descolgada" de la institución donde ejercía. Seguidamente describe los motivos de esa situación y cómo la fue resolviendo. El contexto y el colectivo que rodea a Miriam no le fueron totalmente adversos, si bien al inicio constituyeron un obstáculo, encontró apoyo de autoridades y colegas. La estrategia de esta docente es buscar el apoyo, la orientación en la experiencia de otros. Es la denominada "estrategia social" (Lacey, 1977, citado en Marcelo C.2008), lo que significa que los docentes toman una postura de sumisión estratégica ante las normas establecidas y las prácticas consuetudinarias, para "adaptarse" o bien estar en consonancia con el contexto en que actúan. Son novatos por la escasa experiencia docente, pero son expertos en amoldarse a los 
acontecimientos, cuando se les presenta un obstáculo la respuesta no se hace esperar, es inmediata; es decir, se evidencia la experiencia adquirida en la vida cotidiana, que ahora la "ensayan" en la comunidad educativa. La construcción de la identidad profesional de Miriam, fue realizada con la colaboración del grupo de pertenencia, está marcada por el entorno social y cultural; la representación de su realidad conlleva la marca de sus colegas y directivos además, de su historia personal.

En otro sentido, encontramos docentes principiantes, que reflexionaron sobre las formas de enseñar Historia y los cambios que generaron en las estrategias didácticas, como, por ejemplo, la narración de Zulma:

Mis [dos] primeros años en la docencia ... fui transmisora de una historia tradicional ya realizada, que había que aprender, a lo sumo "comprender como un proceso", pero, a la vez, siempre hice grandes esfuerzos por humanizarla, por superar lo meramente cronológico, y los contenidos según un determinado texto y/o editorial. Me esforcé por seleccionar contenidos para no quedar encerrada en una sola postura. En un juego de ida y vuelta entre lo que viví como alumna no solo en el nivel medio sino en la carrera docente, ya que mi formación profesional estuvo teñida, en algunas cátedras, por esta historia "positivista".

Mis percepciones y sentimientos son, a veces, encontrados y contradictorios, otros claramente esperanzadores y otras veces no tanto. Pero creo firmemente en la pasión por ayudar a construir el conocimiento, a generar autonomía, en la no neutralidad del docente, ni de su postura ni de su pensamiento ni de su aparente objetividad.

Siento pasión por lo que hago, siento que voy creciendo a medida que realizo mi trabajo, siento que el docente transmite en cada clase, mucho más de lo que el mismo docente cree que hace.

Trabajo por la igualdad de oportunidades y si tengo que optar por alguien, lo hago por el más débil.

Zulma, en su estrategia de gestión del aula y en la implementación de métodos de enseñanza para generar un estudiante autónomo, lucha por cambiar la perspectiva de análisis de la Historia, trata de humanizarla, intenta desprenderse de un enfoque tradicional, marcado por la memoria mecánica. En esa lucha tiene sentimientos encontrados, existe una tirantez entre la formación que recibió, por un lado y los cambios que pretende realizar, por el otro. La pasión por la enseñanza y el compromiso constituyen la base que le permite reflexionar, introducir cambios y revisar sus propias concepciones disciplinares.

Observamos en los relatos citados diversas estrategias y actitudes que posibilitan a las principiantes entrevistadas permanecer en la profesión y desarrollar poco a poco el conocimiento de oficio que la experiencia promueve. Aquí, señalamos que las investigaciones acerca de la etapa de iniciación de los noveles revelan también casos deserción ante las dificultades que no pueden resolver. Carlos Marcelo (2008), cita trabajos como los de Cochran-Smith (2004), que indican causas de abandono de la docencia. Entre las más recurrentes mencionan a la insatisfacción debido a los bajos salarios, a los problemas de indisciplina con los estudiantes, a la falta de apoyo de la administración, al trabajo aislado y a las escasas oportunidades para participar en la toma de decisiones.

En relación con las vivencias, altibajos y dificultades de los docentes noveles identificamos una problemática compleja que consideramos debería ser contemplada por las políticas de formación docente y los recorridos curriculares de la formación inicial. Esta última no puede resolver (la formación inicial) sola las necesidades de acompañamiento de quienes transitan la etapa de inserción en la docencia. Se requieren programas de inserción que articulen estrategias de las instituciones formadoras con las instituciones de ejercicio profesional, teniendo en cuenta de que se 
trata de elaborar propuestas específicas, distintas tanto de la formación inicial como de la formación en servicio.

\section{Conclusiones}

El análisis de las narrativas de los "noveles docentes", que desempeñan su labor en cursos de Historia, nos permitió acercarnos a una problemática compleja en la que se entrelazan componentes de la formación universitaria, con creencias, sentimientos y actitudes personales que suscitan decisiones tanto espontáneas como reflexivas.

Los relatos de profesores noveles estudiados proporcionan descripciones de situaciones diversas que viven en los inicios del ejercicio de la profesión. Sin embargo, en todas las narraciones, existe un punto de encuentro cuando reconocen los desafíos y obstáculos que les significa dar los primeros pasos en el proceso de enseñar Historia, en el nivel medio o terciario. Las dificultades que se observan como constantes, se pueden sintetizar en:

- Desconocimiento de la realidad del nivel escolar al egresar de la universidad.

- Una pobre formación pedagógica, a lo que se agrega, una situación que se infiere de las narraciones, que es la disociación entre teoría /práctica.

- Características de los alumnos y su postura de rechazo ante la asignatura Historia, un imaginario colectivo que se concreta al pretender enseñar la disciplina.

- Hallar estrategias para el aula, que motiven a los estudiantes.

- Las exigencias de un contexto institucional desconocido.

Con respecto a aspectos positivos mencionados por los docentes noveles entrevistados en relación con sus experiencias de inserción profesional, encontramos como más significativa a la empatía con colegas y autoridades, lo que les permite daptarse y construir un entramado de relaciones intersubjetivas que contribuye al saber estar y desenvolverse en la nueva situación laboral para ir cimentando su identidad profesional.

Destacamos especialmente, la actitud positiva de los sujetos analizados, quienes a pesar de los obstáculos quieren seguir adelante porque "aman" la profesión, quieren capacitarse y perfeccionarse porque se consideran jóvenes y con potencial para transformar el sistema educativo, como así también existe un punto en común que es, el deseo de formar estudiantes "autónomos", con pensamiento crítico- reflexivo.

Estamos ante la presencia de noveles pero a la vez expertos en adaptarse (Marcelo, 2008) a la realidad de la vida cotidiana en la que deben trabajar, porque como ellos mismos reconocen, egresan desconociéndola, los enfoque teóricos recibidos en las instituciones de formación docente, son nulos o difieren en un grado elevado de la práctica que les toca vivir.

En todos los casos, cuando hacen referencia a la formación docente, destacan que la misma tiene una serie de carencias con respecto a la disociación teoríapráctica, específicamente cuando de asignaturas pedagógicas se trata, existe un quiebre entre la formación en la disciplina y la formación pedagógica; reconocen que manejan muy bien el conocimiento de su ciencia, no así las formas de enseñarlo. Son profesores que están aprendiendo a enseñar con la práctica.

Lo anterior nos plantea la necesidad de introducir cambios en la trayectoria y contenidos de la formación inicial así como estrategias o dispositivos de acompañamiento de los egresados en los inicios de la tarea docente; como es el caso de muchos países e institutos de formación docente. Si este es el camino, habría que preguntarse críticamente qué formación brinda -en este caso la Universidad- para garantizar una inserción profesional satisfactoria cuando otorga el título docente de profesor especialista en una disciplina. 


\section{Referencias bibliográficas}

Alcalá, M. T; Núñez, G. y Demuth, P. (2012). "El conocimiento profesional docente experto. Estudio de caso en la Facultad de Humanidades". Revista Estudios en Ciencias Humanas, $\mathrm{N}^{\circ} 12$, noviembre. Revista digital de Posgrado de la Facultad de Humanidades de la UNNE. 11 páginas.

Alliaud, A. (2004). La biografía escolar en el desempeño profesional de docentes noveles. Buenos Aires: Mimeo (tesis de Doctorado en Educación. Facultad de Filosofía y Letras, UBA).

Álvarez, Z. y Porta, L. (2012). "Caminos de indagación sobre la buena enseñanza: aproximación biográfico-narrativa en educación superior". En Revista de Educación, Año 3, Número 4. Abril. pp. 75-88.

Angulo Rasco, F. (1999). "De la investigación sobre la enseñanza al conocimiento docente. En Pérez Gómez, Ángel; Barquín Ruiz, José y Angulo Rasco, José Félix. (Eds.). Desarrollo profesional del docente. Política, investigación y práctica. Madrid: Akal, 261- 319.

Ball, D. L. y Cohen, D. K. (1999). "Developing practice, developing practitioners: Toward a practice-based theory of professional education". En Sykes, Gary y DarlingHammond, Linda (eds.). Teaching as the learning profession: Handbook of policy and practice. San Francisco: Jossey-Bass. pp. 3-32.

Bolívar, A. (2005). "Conocimiento didáctico del contenido y didácticas específicas". Revista de curriculum y formación del profesorado, 9,2. Universidad de Granada. España. pp. 1-39.

Bransford, J.; Derry, S.; Berliner, D., Hammerness, K., \& Beckett, K. L. (2005). "Theories of learning and their roles in teaching". En Darling-Hammond, Linda \& Bransford, John (eds.). Preparing teachers for a changing world: What teachers should learn and be able to do. San Francisco: Jossey - Bass. pp. 40-87.

Calderhead, J. (1996). "Teachers: beliefs and knowledge", en Berliner, David y Calfee, Robert. (eds.). En Handbook of educational psychology. Nueva York: Macmillan. pp. 709-725.

Clark, C. M. y Peterson, P. L. (1990). "Procesos de pensamiento de los docentes". En Wittrock, Merlin C. La investigación en la enseñanza, III. Buenos Aires: Paidós. pp. 444-539.

Cochran-Smith, M. (2004). Stayers, leavers, lovers and dreamers. Insight about teacher retention. Journal of Teacher Education, 55 (5), 387-392.

Contreras, D. J. (2010). "Ser y saber en la formación didáctica del profesorado: una visión personal". Revista Interuniversitaria de Formación del Profesorado, $N^{\circ} 68$ (24,2). Editorial: Miño y Dávila. Zaragoza. pp. 61-81.

Feiman-Nemser, S. (2001). "From preparation to Practice: Designing a Continuum to Strengthen and Sustain Teaching". Teachers College Record, 103 (6), 1013-1055.

Gorodokin, I. C. (2006). "La formación docente y su relación con la epistemología”. Revista Iberoamericana de Educación, 37 (5), pp. 1-10.

Marcelo, C. (2007). "Empezar con buen pie: inserción a la enseñanza para profesores principiantes". En Docencia N 3 . Diciembre. pp. 27-38.

Marcelo, C. (Coord.) et al.(2008). Profesores principiantes e inserción a la docencia. Buenos Aires: Octaedro.

Marcelo, C. y Vaillant, D. (2009). Desarrollo profeional docente.¿Cómo se aprende a enseñar?. Madrid: Narcea.

Ortega Iglesias, J. M. y Perafán Echeverri, G. A. (2012). "Algunas tendencias en la investigación sobre el conocimiento profesional docente: antecedentes y estado de la cuestión”. En Revista EDUCyT; Vol. 6, Junio-Diciembre. pp. 17-29. 
Perafán, G. A. (2004). La epistemología del profesor sobre su propio conocimiento profesional. Bogotá: Universidad Pedagógica Nacional.

Pérez Gómez, Á. I. (1992). "Enseñanza para la comprensión”. En Gimeno Sacristán, José y Pérez Gómez, Ángel I. Comprender y transformar la enseñanza. Madrid: Morata. pp. 78-114.

Porlán, R. y Rivero, A. (1998) El conocimiento de los profesores. Una propuesta formativa en el área de ciencias. Sevilla: Díada.

Putnam, R. T. y Borko, H. (2000). "El aprendizaje del profesor: implicaciones de las nuevas perspectivas de la cognición". En Biddle, Bruce J.; Good, Thomas L. y Goodson, Ivor F. La enseñanza y los profesores I. La profesión de enseñar. Barcelona: Paidós. pp. 219-309.

Rivas Flores, J. I. (2009). "Narración, conocimiento y realidad. Un cambio de argumento en la investigación educativa". En Rivas Flores, José I. y Herrera Pastor, David. (coord.). Voz y educación. La narrativa como enfoque de interpretación de la realidad. Barcelona: Octaedro. pp. 17-36.

Shulman, L. S. (1989). "Paradigmas y programas de investigación en el estudio de la enseñanza: Una perspectiva contemporánea". En En Wittrock, Merlin C. La investigación en la enseñanza,I. Buenos Aires: Paidós. pp. 9-91.

Veenman, S. (1984). "Perceived Problems of Beginning Teachers". Review of Education Research, 54 (2), 143-178. 